\title{
Ist der Webersche Bürokratietypus ein objektiver Richtigkeitstypus? Anmerkungen zu einer These von Renate Mayntz*
}

\author{
Hartmann Tyrell \\ Universität Bielefeld, Fakultät für Soziologie \\ Universitätsstraße $25, \mathrm{D}-4800$ Bielefeld 1
}

Z u s a m me n f a s u n g: Der Beitrag setzt sich kritisch mit der These von Renate Mayntz auseinander, der Webersche Bürokratıetypus musse als ein ,objektiver Richtigkeitstypus' angesehen werden. Der Verfasser versucht zu zeigen, daß diese These nicht haltbar ist; er tut dies anhand einer genaueren Befassung mit der Bürokratiekonzeption Max Webers, wobei es ihm vor allem darauf ankommt, diese Konzeption starker in den Kontext der Herrschaftssoziologie insgesamt zu rücken. Abschließend bietet der Verfasser einige Überlegungen zu einem adàquateren Verstandnis der Rationalitat der Bürokratie im Sinne Max Webers an.

„,Der entscheidende Grund fur das Vordringen der bürokratischen Organisation war von jeher ihre rein technische Überlegenheit über jede andere Form" (Weber 1964, II: 716; auch 726). Mit solchen und ähnlichen Formulierungen hat Max Weber die moderne Bürokratie und die ihr spezifische Rationalität immer wieder bedacht. Es fallt auf, daß er dabei mit Superlativen keineswegs spart ${ }^{1}$ und zugleich mit immer neuen technisch-instrumentellen Charakterisierungen für die Bürokratie aufwartet: die Rede ist vom ,bürokratischen Mechanismus', vom ,Präzisionsinstrument', von der Bürokratie als ,Apparat", als ,Maschine‘ usw. (1924: 412 ff.; 1964, II: 729; 1971a: 322 f.; vgl. auch Lipp 1978: 9)

\section{Mit dergleichen Metaphern hat Max Weber eine} Interpretation seines Bürokratietypus genährt und kräftig belebt, die dessen superlativische Rationalität primär oder gar ausschließlich ,zweckrational oder im Sinne von ,outputorientierter Effizienz ' verstehen wollte bzw. will. Eine solche Interpretation grassierte in der älteren Organisationssoziologie (Mayntz 1971a; Albrow 1972: $62 \mathrm{ff}$.) und ist nicht weniger beliebt auch in neueren organisationssoziologi-

* Wolfgang Kröpp habe ich für die kritische Durchsicht des Manuskripts zu danken.

1 Die vielleicht bekannteste Stelle sei hier komplett zitiert: „Die rein bureaukratische, also die bureaukratisch-monokratische aktenmäßige Verwaltung ist nach allen Erfahrungen die an Präzision, Stetigkeit, Disziplin, Straffheit und Verläßlichkeit, also: Berechenbarkeit für den Herrn wie für die Interessenten, Intensität und Extensität der Leistung, formal universeller Anwendbarkeit auf alle Aufgaben, rein technisch zum Höchstmaß der Leistung vervollkommenbare, in all diesen Bedeutungen: formal rationalste Form der Herrschaftsausübung" (Weber 1964, I: 164). schen Veröffentlichungen: so nimmt Luhmann (1971: 93) den Bürokratietypus als reines ,Zweckmodell' der Organisation, und anderswo liest man, Weber sei es auf ,eine Struktur optimal zweckrationalen Handelns" (Pfeiffer 1976: 31), auf ein ,einzigartige effizientes System zur Erreichung spezifischer Ziele" (Müller 1973) angekommen. Versteht man den Weberschen Bürokratietypus so, dann hat man es mit der Kritik daran leicht (etwa Luhmann 1971: $94 \mathrm{ff}$.).

Eine besondere Variante der Weber unterschobenen ,Effizienzhypothese' (Kieser/Kubicek 1978: 112) bietet nun die werkimmanente Interpretation des Bürokratietypus, die Renate Mayntz (1971b: $27 \mathrm{ff}$.) in einem häufig zitierten (erstmals 1965 erschienenen) Beitrag angeboten hat. Dieser Beitrag, der Weber gerade gegen ,Mißverständnisse" auf seiten seiner organisationssoziologischen Kritiker in Schutz nehmen wollte, hat der auf ,Zweckrationalität' und ,Effizienz' setzenden Deutung des Bürokratietypus forciert Nahrung gegeben; indem Mayntz eine besondere Version des Weberschen Konzepts des Idealtypus, nämlich die des, objektiven Richtigkeitstypus* für den Typus der ,legalen Herrschaft mit bureaukratischem Verwaltungsstab' (Weber 1964, I: 160 ff.) in Anschlag brachte, dramatisierte sie noch die Zweck-Mittel-Konstellation, der der Bürokratietypus mit seiner langen Reihe von Einzelbestimmungen sich einfugen soll. Denn der ,objektive Richtigkeitstypus", ,dieser eigentlich rationale Idealtypus formuliert, wie ein zweckrationales Verhalten bei richtiger Wahl der Mittel im Hinblick auf ein gegebenes Ziel beschaffen ist" (Mayntz 1971b: 28). Folglich erscheinen dann die von Weber, wie Mayntz (1971b: 28) meint, nach der „Idee der Richtigkeitsrationalität“" ausselek- 
tierten Einzelstücke des Bürokratietypus ganz exklusiv als die ,objektiv richtigen' Mittel ,für die dauerhafte und technisch effiziente $A u s$ übung legaler Herrschaft" (Mayntz 1971b: 30). Sieht man die Dinge so, dann ist es nur konsequent, den Bürokratietypus auch zum ,formellen Sollschema' zu erklären und ihn sodann in die nächste Nähe des, präskriptiven Modells der klassischen Organisationslehre' zu rücken (Mayntz 1971b: 29; ebenso Luhmann 1971: 93).

Dieser von Mayntz ohne größeren Begründungsaufwand eingefuhrten Deutung ist bislang seitens der Weberexegese merkwürdigerweise nicht widersprochen worden; ganz im Gegenteil: man hat sie teils unbeanstandet passieren lassen, teils einfach ,nachgebetet ${ }^{\circ}$. Dabei drängen sich bei näherem Zusehen die Einwände geradezu auf, die gegen die von Renate Mayntz vorgenommene Identifizierung des Weberschen Bürokratietypus als eines rationalen Richtigkeitstypus geltend zu machen sind. Sie sollen hier im weiteren entwickelt werden.

Max Weber wirft das Problem der ,Richtigkeitsrationalität" in dem Aufsatz, Uber einige Kategorien der verstehenden Soziologie“ (1973: 427 ff. $)^{3}$ dort auf, wo er im Blick auf ein

2 Das erstere gilt etwa für die aufwendige neue Arbeit von Prewo (1979: 87, Anm. 2, 113, Anm. 45, 484, Anm. 77), der den Mayntzschen Aufsatz mehrfach gegen die ältere Organisationssoziologie ins Feld führt. Zu den ,Nachbetern' zählen Schluchter 1972: 122, Müller 1973: 723 f., Gabriel 1974: 352, Pfeiffer 1976: 30 f., Gabriel 1979: 31. Überhaupt wird auf den Aufsatz von Mayntz (1971b) immer wieder Bezug genommen, wo man Weber gegen ,Mißverständnisse* verteidigen möchte; etwa Grunow/Hegner 1977: 52; auch Kieser/Kubicek 1978: $110 \mathrm{f}$..

3 Max Weber verwendet, soweit ich sehe, die Termini ,Richtigkeitsrationalität" und ,Richtigkeitstypus" nur in diesem, nun allerdings höchst wichtigen Aufsatz von 1913; das Problem aber taucht später durchaus noch auf, so im ,Wertfreiheits'-Aufsatz von 1917 (1973: $525 \mathrm{ff}$.) und in den ,Soziologischen Grundbegriffen' $(1964, \mathrm{I}: 4 \mathrm{f}$ ) , welch letztere ja bekanntlich auf dem, Kategorien'-Aufsatz fußen. Allerdings gibt es zu denken, daß auch das Konzept der ,Richtigkeitsrationalität' und des ,Richtigkeitstypus' den ,mehrfachen Veränderungen " in der Terminologie, von denen eingangs der ,Soziologischen Grundbegriffe* (1964, I: 3) ausdrücklich die Rede ist, zum Opfer gefallen ist. Auf den ,Kategorien-Aufsatz verweist auch Mayntz (1971b: 34, Anm. 3); den Tatbe- ,streng zweckrational orientiertes Handeln ' die spezifischen .Evidenzen` und ,Verstehensleistungen' der Soziologie von denen der Psychologie abhebt (432 ff.). Dabei trennt er scharf zwischen einem, subjektiv zweckrational orientierten Handeln', wie es etwa auch ein mit den Mitteln der Magie operierendes zweckorientiertes Handeln sein kann, und einem ,objektiv zweckrationalen“, nämlich ,,am objektiv Gültigen“" orientierten, nach wissenschaftlichen Erfahrungsregeln die, richtigen 'Mittel einsetzenden Handeln (433 f.). ( $\mathrm{DaB}$ beides faktisch koinzidieren kann, versteht sich.) Nach Weber kommen nun Soziologie und Geschichtswissenschaft gar nicht umhin, Typen des, richtigkeitsrationalen Handelns', abgekürzt auch ,Richtigkeitstypen', zu entwerfen, um sich den ihnen gemäßen ,verstehenden' Zugang zur empirischen Wirklichkeit des sozialen Handelns zu verschaffen.

Hier ist nun zunachst wichtig, daß sich Weber in der einschlagigen Passage, die einzig das Thema der Richtigkeitsrationalität explizit angeht, sich in aller Deutlichkeit auf einen Typus des Handelns (mit spezifischer Orientierung und Ablauflogik) bezieht ${ }^{4}$. Ich mochte mich hier nun nicht mit der Dichotomieproble. matik von ,handlungstheoretischer versus strukturtheoretischer Begrifflichkeit' belasten, wie sie Bader u.a. (1976; auch Berger 1978) mit

stand, daß der Richtigkeitstypus nur hier abgehandelt wird, uberspielt sie mit der Floskel, dieser Typus werde hier ,am klarsten ... dargestellt". Im übrigen ist die Weberexegese auf das Problem der Richtigkeitsrationalität (als Spezialfall von $Z$ weckrationalität) kaum irgendwo naher eingegangen; auch Prewo (1979: 256, 367, Anm. 167, 525) erwähnt es nur am Rande.

4 Der ,Kategorien'-Aufsatz fuhrt im ersten Abschnitt das Handlungskonzept als Kernstück der, verstehenden Soziologie، ein (Weber 1973: 427 ff.) und grenzt diese dann im zweiten Abschnitt (432 ff.) gegen die Psychologie ab; eben dabei bedient sich Weber entscheidend des Konzepts der Richtigkeitsrationalität des Handelns; denn ein Handeln ist in seinem Ablauf fur ihn umso, evidenter' und rationaler verstehbar, je eindeutiger es ,,dem Typus der Richtigkeitsrationalität entsprechend orientiert ist", und desto weniger bedarf es dazu ,psychologischer Erwägungen' (432). Damit sollte aber deutlich sein: das Thema der Richtigkeitsrationalitát gehört bei Weber primar in den handlungstheoretischen Kontext und nicht, wie Mayntz (1971b: 34, Anm. 3) nahelegen möchte, in den Zusammenhang der „Darstellung des Idealtypus". 
Bezug auf Weber allenthalben geltend machen; aber so viel steht außer Zweifel: der Webersche Bürokratietypus hat mit seiner langen Reihe von Einzelbestimmungen nicht das konstruierte Ablaufmodell eines die ,richtigen Mittel' verwendenden, zweckorientierten Handelns, das von da aus verstehbar Subjekten zurechenbar wäre, zum Gegenstand.

Die Dinge liegen komplizierter. Einerseits nämlich geht es auch bei der Weberschen Bürokratie ganz wesentlich um eine bestimmte Typik des Handelns; es geht darum, daß das, Verwaltungshandeln" auf Dauer und „, bei einer angebbaren Vielheit von Menschen" (1964, I: 38), also ,sozial generalisiert", in spezifischer Weise diszipliniert, regelgebunden, strikt sachorientiert, ,unpersönlich', präzise und berechenbar abläuft und ablaufen kann. Eben darin, im ,Gehorsamshandeln", das der spezifisch burokratische Verwaltungsstab mit besonderer Extensität und Intensität erbringt, zeigt sich der Bürokratietypus je als Herrschaftstypus ${ }^{5}$. Zugleich gilt es zu sehen, daß das bürokratische Handeln als solches, da es primär ,konditional programmiert ${ }^{\star}$ ist, typisch nicht zweckrational verfährt; es operiert ,streng formalistisch nach rationalen Regeln und - wo diese versagen - nach sachlichen" Zweckmäßigkeitsgesichtspunkten" (1973: 476). Hinter den Regeln selbst allerdings mögen durchaus Zwecke stehen, und insofern wiederum ist das bürokratische Verwaltungshandeln in Zweck-Mittel-Relationen eingespannt.

Andererseits aber erschöpft sich der Bürokratietypus, wie ihn Weber verschiedentlich im Kontext der Herrschaftssoziologie entwickelt hat ${ }^{6}$,

5 Auf den Weberschen Herrschaftsbegriff bin ich an anderer Stelle eingegangen; siehe Tyrell 1980.

6 Zur Werkgeschichte der Herrschaftssoziologie siehe Roth 1968: LXXXII f., Mommsen 1974a: $202 \mathrm{ff}$., 275 f., Anm. 58, auch 1974b: 16 f., 72 ff.. Seine ,Herrschaftstypologie' und ,-soziologie' hat Weber mit jeweils unterschiedlicher ,Breite und Dichte* im wesentlichen an drei Stellen ausgearbeitet (1964, 1: 157 ff., II: 692 ff., 1973: 475 ff.), wobei der Typus der rational-legalen Herrschaft (und dabei primär der der bürokratischen Herrschaft) jeweils als erster abgehandelt wird. Zum Problem der Anordnung der Typen mit unterschiedlichen Akzenten Winckelmann 1964: 46, Anm. 56, und Mommsen 1974a: 128. Wesentliches zur Bürokratiethematik findet sich natürlich auch in Webers politischen Schriften; siehe vor allem 1971a, 1971b, ferner Beetham 1974: $63 \mathrm{ff}$. . keineswegs in der Charakterisierung der Typik eines besonderen Verwaltungshandelns. Der Typus benennt daruberhinaus - etwa mit den von Weber besonders wichtig genommenen Bestimmungen der ,völligen Trennung ${ }^{6}$ des Beamten ,,von den Verwaltungsmitteln" und des Fehlens jeder "Appropriation der Amtsstelle" (1964, I: 163) - strukturelle Rahmenbedingungen dafür, $\mathrm{daß}$ das ,Verwaltungshandeln ${ }^{*}$ in der genannten Art ablaufen kann. Hier sind ,die handlungstheoretischen Begriffe . . mit komplexeren Strukturbegriffen verkettet" (Seyfarth 1979: 156). Weber (1964, II: 737 f.) selbst spricht hier von "Strukturprinzipien“ oder „Organisationsprinzipien“ der Herrschaft bzw. Verwaltung, etwa vom „Prinzip der festen ... Kompetenzen“ oder vom „Prinzip der Amtshierarchie und des Instanzenzuges" (1964, II: 703). Es versteht sich aber fast von selbst, daß diese Strukturkomponenten des Typus sich der spezifischen Rationalität eines die richtigen Mittel bei gegebenen Zwecken verwendenden Handelns nicht fügen, und schon das verbietet es, den Bürokratietypus als, objektiven Richtigkeitstypus' zu nehmen.

Sieht man aber einmal von der Gebundenheit des Weberschen Konzepts der ,Richtigkeitsrationalität" an die Handlungsebene ab, so ist darüberhinaus Renate Mayntz' (1972b) Interpretation des Bürokratietypus gerade hinsichtlich der angegebenen Zweck-Mittel-Relation ,schief'. Sie behauptet einerseits zurecht, ,Eigenart und . . Inhalt manifester Verbandsziele" spielten bei Weber im Gegensatz zur modernen Organisationssoziologie ,keine besondere Rolle“(28); in der Tat: gerade für die moderne Bürokratie betont Weber den ,Vielzweckcharakter', ja: ,formal universelle Anwendbarkeit auf alle Aufgaben" (1964, I: 164). Andererseits stellt Mayntz an gleicher Stelle (1972b: 28) - ebenso zu Recht - fest, jeder ,objektive Richtigkeitstypus“ setze ,ein gegebenes Ziel" voraus, auf das hin es allein ,richtige Mittel' geben kann. Ein solches inhaltlich instruktives Ziel ist nun aber „die wirksamste Herrschaftsausübung" als solche - einzig spezifiziert durch den Verweis auf den Kontext der ,legalen Herrschaft" (Mayntz 1971b: 28) - doch wohl kaum; ohne Angaben hinsichtlich der Quantität der Beherrschten (Weber 1964, II: 713 ff.), der Qualität und Komplexität des ,Aufgabenkreises‘ und der Intensität der Herrschaftspraxis (1964, II: 715 f.), ohne Spezifizierungen auch hinsichtlich der 
ökonomischen oder religiösen Umweltlage ${ }^{7}$, läßt sich die hohere Rationalität der Bürokratie als ,Mittel der Herrschaftsausübung' nicht ,deduzieren“, läßt sich nicht einmal der Rationalitätsvorsprung des bürokratischen Verwaltungsstabs gegenüber ,unmittelbar demokratischer Verwaltung' $\left(1964\right.$, Il: 697) klären ${ }^{8}$.

Und weiter: ,die einzelnen Merkmale des Weberschen Bürokratiebegriffs" sind, hält man sich an die Auffassung von Renate Mayntz (1971b: 28), ,die Antwort auf die Frage . . . , wie der Herrschaftsstab im Falle legaler Herrschaft beschaffen sein müsse, um die wirksamste Herrschaftsausübung zu gewährleisten ". Interpretiert man den Bürokratietypus auf dieser Linie oder nimmt ihn als reines ,Zweckmodell der Organisation", so unterstellt man damit hinsichtlich seiner einzelnen Bestimmungen, daß diese von Weber (einigermaßen vollständig) abgeleitet, ermittelt und zusammengestellt seien exklusiv unter dem Gesichtspunkt ihrer Mittelqualität im Hinblick auf den vorausgesetzten Herrschaftszweck; man unterstellt weiter, daß Weber unterstellt, daß sie sich zusammenfügen zu einem relativ geschlossenen und ausschlieblich zweckorientierten System von logisch und systematisch kohärenten Merkmalen. Damit sind zwei Fragen angeschnitten, die gewissermaBen als Rückfragen an Max Weber zu richten sind und die es nunmehr näher zu behandeln gilt: Zum einen: unter welchen Fragestellungen und Gesichtspunkten hat Weber die einzelnen Komponenten seines Bürokratietypus bestimmt und ausgewählt? War dabei wirklich die ,Idee der Richtigkeitsrationalität" leitend? Zum anderen: in welcher Beziehung stehen die einzelnen

7 Hier hat Max Weber (1964, II: $709 \mathrm{ff}$ ) vor allem die ,Geldwirtschaft' im Auge; siehe aber auch das Kapitel über ,politische und hierokratische Herrschaft $t^{\circ}$ (1964, II: 874 ff.). Im übrigen macht Renate Mayntz (1971b: 30) weiter unten mit Bezug auf die gesellschaftliche Umwelt dann noch eine weitere, zudem historisch akzentuierte Einschrảnkung, indem sie die ,Zweckrationalität ${ }^{*}$ der Bürokratie an die ,,Bedingungen des wirtschaftlich entwickelten und politisch zentralisierten Nationalstaates" bindet.

8 Zudem ist, wie Weber (etwa 1964, II: 712) sehr genau sieht, die patrimoniale Herrschaft, die mit ,persönlich Abhängigen', mit Sklaven, Unfreien, Landfremden usw. operiert, hinsichtlich der Bedingungslosigkeit von deren Unterwerfung unter den Herrn der Bürokratie deutlich überlegen.
Komponenten zueinander? Fügen sie sich wirklich als objektiv richtige Mittel zu einem ,Modell hóchster Zweckmäßigkeit" (Mayntz 1971b: 28) zusammen?

Nimmt man sich die Details des Weberschen Bürokratietypus vor ${ }^{9}$, so zeigt sich schnell, daß sie zusammengestellt und arrangiert sind unter ganz bestimmten, relativ leicht rekonstruierbaren Relevanz- und Problemgesichtspunkten (Bendix 1965: 436). Dabei sollte man sich aber bewußt halten, daß gerade der Webersche Bürokratietypus keineswegs eine, creatio ex nihilo" ist, sondern in starkem Maße anschließen konnte vor allem an die verwaltungsgeschichtlichen Studien Gustav Schmollers (1894; 1898), die insbesondere die preußische Bürokratie und Staatsverwaltung im Visier hatten ${ }^{10}$. Dementsprechend hält sich die Auswahl der Merkmale

9 Dabei muß man die Bauprinzipien der Weberschen Herrschaftstypologie im Auge haben. Diese Typologie differenziert primar nach drei, ,in ganz reiner Form, nur dre1" (1973: 475) heterogenen Mustern der ,Legitimitätsgeltung“ der Herrschaft (etwa 1964, 1: 159). Sie differenziert sekundar, indem sie die Legitimitàtstypen verknupft ,mit einer grundverschiedenen soziologischen Struktur des Verwaltungsstabs und der Verwaltungsmittel" (1973: 475). Das zielt insbesondere auf die Appropriationsproblematik und die unter diesem Gesichtspunkt innerhalb des Typs der, traditionalen Herrschaft' vorgenommene Unterscheidung von ,patrimonialer" und ,ständisch-feudaler' Verwaltungsstruktur (etwa 1973: 478 ff.); der Appropriationsgesichtspunkt ist naturlich auch für den Burokratietypus zentral wichtig und bringt ihn hier auf eine Linie mit dem Patrimonialismus. Die Bauweise der Herrschaftstypologie ist besonders transparent bei Weber (1973: $475 \mathrm{ff}$.), auch wenn die Terminologie hier leicht variiert ist. Siehe auch Abramowski 1966; 122 ff., 127 ff.; ferner Bendix 1964: $220 \mathrm{ff}$, sowie -- besonders differenziert - Zingerle 1972: 20 ff., 46 ff..

10 Darauf hat mit Martin Albrow (1972: $59 \mathrm{ff}$.) ein Englander aufmerksam gemacht. Die Studien Schmollers stehen im engen Zusammenhang mit der von ihm besorgten Herausgabe der Acta Borussica, der preußischen Staats- und Verwaltungsakten des 18. Jahrhunderts. Auch auf Otto Hintze (etwa 1964 - Vortrag von 1911), den Schuler und Mitarbeiter Schmollers an den Acta Borussica, ware hinzuweisen. Im ubrigen stand Weber innerhalb des Vereins für Sozialpolitik (auch) hinsichtlich der Einschätzung der politischen Rolle der Bürokratie im deutlichen Gegensatz zu Schmoller und der ,älteren Generation'; siehe Lindenlaub 1967: 238 ff., 292 ff., 393 ff.; ferner Beetham 1974: $63 \mathrm{ff}$., 
des Weberschen Bürokratietypus einerseits, wie Renate Mayntz (1971b: 28, 30) selbst sieht, an die zeitgenössische preußisch-deutsche Verwaltungsorganisation der Jahrhundertwende: in deren Kontext ist die strukturelle Relevanz etwa der ,festen Amtshierarchie', der ,festen Amtskompetenzen“, des Reglements oder der ,Aktenmäßigkeit der Verwaltung' für das soziologische Auge schon ,auf den ersten Blick' evident. Andererseits sind die Merkmale zusammengestellt nach abstrahierenden und tendenziell ,theoretischen' Gesichtspunkten, die ihrerseits dem Vergleich und der Kontrastienung der modernen Bürokratie mit historisch älteren bzw. strukturell alternativen Verwaltungsformen der Moderne $^{11}$ abgewonnen sind; die strukturelle Relevanz erschließt sich hier erst über das komparative Vorgehen. Solche Gesichtspunkte und Leitfragen, die sich quer durch die Webersche Herrschaftstypologie ziehen, sind u.a.: die Frage nach der ,Rekrutierung' und sozialen Herkunft des Beamtenpersonals und dem Modus seiner Bestallung (,Ernennung von oben' oder Wahl oder Kauf usw.), die Frage nach dem Verhältnis von. Amt und Person (insbesondere: Appropriation oder ,Trennung'), die Frage nach der wirtschaftlichen Versorgung der Beamten und ihrem Verhältnis zu den Verwaltungsmitteln, ferner die Frage nach dem spezifischen Sozialprestige und damit eng verbunden: nach der jeweiligen kulturgetragenen Gehorsamsmentalität (Pietät oder Dienertreue oder Amtsdisziplin) der Beamten. All diese Fragen sieht und analysiert Weber vor allem im Hinblick auf die Problematik ihrer Bedeutung für die Machtstruktur von Herrschaftsverbänden ${ }^{12}$. Zugleich korreliert er seine historischen Analysen der jeweiligen ,Struktur des Verwaltungsstabs und der Verwaltungsmittel' (1973: 475) mit immer neu gestellten Fragen nach deren struktureller Kompatibi-

11 Es geht mit Bader et al. (1976: 454), um einen doppelten Vergleich: a) den universal-historischen Vergleich mit vorbürokratischen, traditionalen Formen der Herrschaft; b) den Vergleich mit der ,Honoratioren-Verwaltung' einerseits, demokratischen Organisationsformen andererseits". Siehe auch Schluchter 1972: 120; ferner Gabriel 1976: $302 \mathrm{f}$.

12 So ist nach Weber (1964, I: 196) „die historische Realität auch ein steter, meist latenter Kampf zwischen Herrn und Verwaltungsstab um Appropriation oder Expropriation des einen oder des anderen". Vgl. hier im Hinblick auf China Zingerle 1972: 54 ff.. lität bzw. Inkompatibilität mit einer, straffen Durchhierarchisierung ' und ausgeprägten ,Betriebsdisziplin“; Weber fragt weiter nach der Zugänglichkeit von historischen Verwaltungsstrukturen für eine ,abstrakte Reglementierung' des Verwaltungshandelns, ebenso für Gesichtspunkte einer sachlich angeleiteten Arbeitsteilung und fachlichen Qualifikation und Einschulung der Beamten. Schließlich fragt er immer wieder nach der ,Berechenbarkeit' und Kalkulierbarkeit des Verwaltungshandelns für die Umwelt, vor allem: ,den Herrn und die Interessenten".

Damit aber ist deutlich: der Webersche Bürokratietypus ist vor dem Hintergrund dieses herrschaftssoziologischen Problemarsenals entworfen nach bestimmten sachlichen Relevanzgesichtspunkten, die fuir Weber - sei es im Blick auf die preußische Bürokratie, sei es unter komparativer Perspektive - , auf der Hand lagen". Hier werden nicht Mittel aus bestimmten Zielvorgaben, theoretisch deduziert ${ }^{13}$, sondern hier wird soziologisch-universalhistorisch ,nach allen Erfahrungen" (Weber 1964, I: 164) geurteilt. Und erst recht liegt hier der Gedanke an eine ,systemische Geschlossenheit oder gar Vollständigkeit des Typus fern. Die wenigstens, an den Rändern' relativ lockere Kompositionsweise wird im ubrigen schon daran deutlich, daß von den drei ,Hauptfassungen" des Bürorkratietypus (1964, I: 160 ff.; II: 703 ff.; 1973: 475 ff.) keine mit den jeweils anderen völlig deckungsgleich ist. Zudem bekommt zumal die spezifische Rationalität des Typus je nach Vergleichsgesichtspunkt bei Weber teilweise recht heterogene Akzente. Damit ist die andere Frage nach dem Zusammenhang, in dem die einzelnen Elemente des Weberschen Bürokratietypus miteinander stehen, schon angegangen. Die Antwort muß hier lau. ten: die Einzelmerkmale sind, soweit sie nicht auf die Bestimmung der spezifischen Typik des bürokratischen Verwaltungshandelns bezogen

13 Das räumt Mayntz (1971b: 28) denn auch selbst ein, wenn sie ausdrücklich, zugibt", Weber habe ,seine Elemente nicht explizit und dem Leser augenfällig durch theoretische Ableitung gewonnen". Soll damit behauptet werden, Weber habe die Deduktionen ,im Verborgenen vorgenommen? Wohl kaum! Mayntz' letztes Wort in dieser Sache ist dann, Weber habe ,die Idee der Richtigkeitsrationalität ... gleichsam nur als Selektionsprinzip bei der Auswahl historisch-empirischer Elemente benutzt" $(28)$. 
sind, zusammengestellt nach Gesichtspunkten der jeweiligen strukturellen Kompatibilität vor allem mit dieser Typik des Handelns; keineswegs aber gehen sie als ein kohärenter Katalog von abstrakt deduzierten, richtigen Mitteln“ im Hinblick auf einen übergeordneten Zweck zusammen.

Die Webersche Soziologie ist voll von Kompatibilitätserwägungen; Weber selbst spricht gern von ,Wahlverwandtschaft ${ }^{*}$ oder von ,Adäquanzbeziehungen', wobei es darum geht, ,ob und wie stark" verschiedene soziale Strukturgegebenheiten ,sich gegenseitig in ihrem Bestande beguinstigen oder umgekehrt einander hemmen oder ausschließen" (1964, I: 259). Von Fragestellungen, Überlegungen und Argumenten dieses Typs ist, wie zuvor schon angedeutet, auch die Herrschaftssoziologie durchsetzt. Man sehe sich nur die erfahrungsgesättigten Argumente Webers etwa gegen das Wahlbeamtentum an, das er für inkompatibel ansieht mit strikter Amtsdisziplin, Sachlichkeit und gehobener Fachqualifikation der Amtsausübung (1964, I: 199 f.; II: 706 f.); der ,reine Typus' (mit seinem Prinzip der Amtshierarchie), erfordert ${ }^{*}$ den von oben ernannten Beamten. Oder man sehe sich Webers Überlegungen zur Koexistenz von Bürokratie und Geldwirtschaft an (1964, II: 708 f., 709 ff.), wo die strukturellen Voraussetzungen und Vorzuge der Geldentlohnung der Beamten vor einem historisch weit gespannten (und eine Reihe von äquivalenten Varianten durchspielenden) Hintergrund entwickelt werden. Nicht abstrakte Zweck-Mittel-Kalkulationen sind es, die die einzelnen Elemente des Bürokratietypus sachlich zusammenführen, sondern aus reichhaltiger Empirie heraus angestellte, theoretisch zudem höchst anregende Erwägungen über Kompatibilitäten und Inkompatibilitäten.

Schließlich: Renate Mayntz (1971b: 32 f.) betont selbst die ,Verankerung' des Bürokratietypus im weiteren Kontext der Weberschen Herrschaftssoziologie, und zurecht hält sie der Organisationssoziologie vor, das gänzlich ausgeblendet, also die Bürokratie völlig isoliert genommen zu haben ${ }^{14}$. Fragt man nun, welcher

14 Allerdings muß man wenigstens der amerikanischen Organisationssoziologie der $40 \mathrm{er}, 50 \mathrm{er}$ und $60 \mathrm{er}$ Jahre mit Mayntz (1971b, 33) erschwerte Rezeptionsbedingungen zugutehalten. Immerhin aber hat
Art die Einbindung des Bürokratietypus in die Herrschaftstypologie ist, so ergibt sich, wie schon angedeutet: auf der ganzen Linie ist Max Webers Burokratietypus konzipiert als ein spezifisch rationaler - Kontrasttypus. "None of Weber's 'ideal types' are defined absolutely but only in terms of contrast with others; it is part of their logic" (Beetham 1974: 90. Anm. 21). Das gilt, wie man schon an der spezifisch ,negativen“ Fassung verschiedener seiner Merkmale sieht ${ }^{15}$, gerade für den Bürokratietypus; hier versteht sich kaum eines der Merkmale ,von selbst", also ohne Mitkenntnisnahme der Gegentypen $^{16}$; allenthalben kommt es Weber auf die Differenz zu diesen gerade an. Und den kontrastiven Zuschnitt des Bürokratietypus

Reinhard Bendix (1947: 497) schon recht fruh darauf hingewiesen, daß die ,Rationalisierung' der Bürokratie kontrastiv zu verstehen ist. Die ganz isolierte Kenntnisnahme und Rezeption des Weberschen Burokratietypus tadelt aber auch Diamant (1962: 61, 66 ff.). Unentschuldbar ,beschrankt ist in diesem Punkt aber Muller (1973), der uberdies - 1973 in einer deutschen Zeitschrift ,Wirtschaft und Gesellschaft t $^{\star}$ nach der alteren und bloß teilweisen, von Henderson und Parsons besorgten amerikanischen Übersetzung von $1947 \mathrm{zi}$ tiert.

15 Negativ gefaßt in diesem Sinne sind: die Bestimmungen der ,Trennung der Beamten von den Amtsstellen und Verwaltungsmitteln, das Moment, daß die Beamten ,angestellt (nicht: gewahlt) sind“" (1964, I: 162), in der Sache weiterhin etwa deren Kennzeichnung als ,persönlich frei“" (1964, I: 162), namlich: nicht unfrei. All diese Bestimmungen sind verständlich nur als Negierung eines Kontrastty pus.

16 Das gilt noch für die auf den ersten Blick so selbstverstandlich scheinende Kennzeichnung der Burokratie als ,Betrieb' und spezieller: ,Behörde‘ (1964, I: $161 ;$ Il: 702; 1973: 475), die auf die Burokratie als eine Einrichtung von Dauer und ihre Befassung mit der ,kontinuierlichen' Abwicklung von Amtsgeschàften zielt. Das aber ist bei Weber zu sehen auf dem Hintergrund des ganz generellen, evolutionstheoretischen" Befunds einer ,Stufenleiter der Entwicklung von der Gelegenheitsvergesellschaftung ausgehend und fortschreitend zum perennierenden ,Gebilde" ", wie sie historisch immer wieder zu finden sei (1973: 451) und wie sie Weber auch immer wieder thematisiert hat. So spricht er mit Blick auf die Anfange der ,Patrimonialbürokratie* vom ,"Zustand des ,Gelegenheits-Beamten" " und auf höherem Entwicklungsniveau - von ,fortgeschrittener Perennität und Stereotypisierung der Ämter" (1964, II: 760). Immer ist am Weberschen Betriebsbegriff (1964, 1: 37) der evolutionäre Kontrast zur Gelegenheitsvergesellschaftung mitgedacht. 
gilt es gerade dann im Auge zu haben, wenn man auf die Superlative abhebt, die Weber immer wieder (etwa 1964, I: 164; ebenso II: 716 f.; 1924: 413) für die Bürokratie bereithält. Diese Superlative von der höchsten Rationalität, die die Bürokratie repräsentiert, sind eben: Komparative; ihr Vergleichsfeld sind immer nur jene, alternativen Formen von Verwaltungsstäben", also, wie gesagt, vor allem Patrimonialismus, Feudalismus, demokratische und Honoratiorenverwaltung.

Gerade wenn man die so geartete Anbindung des Bürokratietypus an die anderen Herrschaftsund Verwaltungstypen bei Weber sieht, gerät nun aber die Berechtigung, diesen als Richtigkeitstypus zu nehmen, vollends ins Wanken. Denn man ist im Falle einer solchen Interpretation ja gezwungen, dem Bürokratietypus innerhalb der Herrschaftstypologie einen unbedingten Sonderstatus zuzusprechen, und muißte zudem angeben können, welche ganz andere Art von Idealtypus dann die anderen Herrschaftstypen repräsentieren, und vor allem: nach welchen ganz andersartigen Konstruktionsprinzipien diese, die ja eben nicht ,Richtigkeitstypen sein können, gebaut sein sollen. Die Deutung des Bürokratietypus als ,Richtigkeitstypus" macht es unumgänglich, die Webersche Herrschaftssoziologie zu zerschneiden; auch sie, isoliert" den Bürokratietypus. Eben dazu aber gibt die Webersche Herrschaftstypologie mit ihrem Bauprinzip der kontrastiven Verweisungen und komparativen Querverbindungen m.E. an keiner Stelle irgendwelchen Anlaß. Und daß Weber die Sonderkennzeichnung des Bürokratietypus als eines ,Richtigkeitstypus' selbst explizit auch nirgendwo vornimmt, versteht sich dann fast schon von selbst.

In anderer Hinsicht allerdings wäre durchaus zuzugestehen, daß dem reinen Typus der ,legalen Herrschaft mit bureaukratischem Verwaltungsstab" innerhalb der Weberschen Herrschaftssoziologie gewissermaßen ein Sonderstatus zukommt. Er ist hier der spezifisch rationale Typus, und als solcher ist er -- vor universalhistorischem Hintergrund - ein individualisierender Typus, der als Wirklichkeit, auf die er bezogen ist, die spezifisch okzidentale Strukturform der Herrschaft im Visier hat. Unbedingt ist auch die ,rationale Bürokratie ${ }^{*}$ als ,Entwicklungsprodukt ' jenes universalhistorischen Prozesses anzusehen, der ,,auf dem Boden des Okzidents, und nur hier, Kulturerscheinungen" eines umfassenden Rationalismus hat entstehen lassen (Weber 1920: 1); unbedingt kommt der ,,an rationalen, gesatzten Regeln .. . orientierten Verwaltung durch Fachbeamte" eine ganz entscheidende Stellung innerhalb des ,spezifisch gearteten ,Rationalismus' der okzidentalen Kultur" zu (Weber 1920: 3 f., 11) Zwar hat Weber deutlicher noch am Kapitalismusbegriff demonstriert ${ }^{17}$, wie sehr seine Strategie der Begriffsbildung und im engsten $\mathrm{Zu}$ sammenhang damit sein allenthalben praktiziertes komparativ-kontrastives Verfahren von einem individualisierenden Erkenntnisinteresse getragen sind ${ }^{18}$; es kann aber nicht der geringste Zweifel sein, daß das ebenso für das Bürokratiekonzept, das als solches unter Webers Zeitgenossen nicht strittig war, gilt (vgl. etwa Weber 1920: 3 f.). Karl Gabriel (1979: 29 ff.) hat zu Recht darauf aufmerksam gemacht, daß man die Bürokratie bei Weber in engem Zusammenhang sehen muß mit der ,kapitalistisch-rationalen Arbeitsorganisation" (Weber 1920: 9); eben diese aber war für Weber (1920: 5, Anm. 1) in ökonomischer Hinsicht gerade, das Spezifische des Okzidentes". Vollends aber wird daran deutlich, wie fern der, wirklichkeitswissenschaftlich' intendierte Bürokratiebegriff Webers jenem abstrakt-ahistorischen, analytisch-deduktiv genommenen ,Modell höchster Zweckmäßigkeit" ist, als das ihn Mayntz (1971b: 28) identifizieren wollte; zugleich zeigt sich, wie sehr Renate Mayntz die Mißverständnisse, gegen die sie Weber in Schutz nehmen wollte, eher noch potenziert hat.

Eine Bemerkung sei hier noch angefuigt zu der verwandten Interpretation, die jene höchste $\mathrm{Ra}$ -

17 Hier stand er hinsichtlich der Begriffsbildung aber eben auch in handfestem Dissens zu verschiedenen zeitgenössischen Kritikern seiner Protestantismusstudie. Vgl. nur Weber (1920: 4 ff., insbes. 4 f., Anm. 1), wo die Intention, ,das Spezifische des okzidentalen Kapitalismus gegeniber anderen Formen herauszuarbeiten", nachdrücklich artikuliert wird.

18 ,Zweck der idealtypischen Begriffsbildung ist es überall, nicht das Gattungsmäßige, sondern umgekehrt, die Eigenart von Kulturerscheinungen scharf zum Bewußtsein zu bringen" (Weber 1973: 202). Das führt an die Kernintentionen der Weberschen Konzeption von ,Wirklichkeitswissenschaft" heran. Vgl. dazu Weiß 1975: $20 \mathrm{ff}$., $65 \mathrm{ff}$., sowie jetzt Kröpp 1979, insbes. $10 \mathrm{ff}, 92 \mathrm{ff}$; ferner auch Prewo 1979: $95 \mathrm{ff}$. 
tionalität des Bürokratietypus im Sinne ,maximaler Effizienz' mißversteht. Gerade hier hat Albrow (1972: $71 \mathrm{ff}$.$) mit guten Argumenten$ widersprochen ${ }^{19}$. Aber Albrow (1972: 77) überzieht doch, wenn er abschließend behauptet, daß die Webersche Bürokratietheorie ,Probleme der Leistungsfähigkeit und Wirtschaftlichkeit völlig ignorierte". Daß Weber das nicht tat, zeigt sich schon daran, daß er mehrfach (so 1924: 413; 1964, II: 716 f.) die Bürokratie im Unterschied zur Honoratiorenverwaltung unter Kostengesichtspunkten explizit ,billig' genannt hat; hierher gehört auch, daß er immer wieder die vergleichsweise ,Schleunigkeit", das spezifische - zumal durch das forcierte Tempo des modernen Kapitalismus unter ,Zeitdruck“ gesetzte - ,Reaktionstempo“ der Bürokratie betont hat (besonders deutlich: 1964, II: 717). Gleichwohl bleibt es richtig, daß die spezifische Rationalität des Bürokratietypus primär nicht auf ,outputorientierte Effizienz‘ zielt.

Was aber macht dann die besondere Rationalität der Bürokratie im Weberschen Sinne aus? Bevor man sich dieser Frage zuwendet, tut man gut daran, sich bewußt zu machen, daß Max Weber selbst die ,Vieldeutigkeit' des Begriffs der, Rationalität ' und der ,Rationalisierung" immer wieder ausdrücklich betont hat (etwa 1920: 11 f., 35, 62, 265 f., $537 ; 1964$, I: 22). Aber mehr noch: die Vieldeutigkeit des Rationalen war ihm ersichtlich eine Angelegenheit des höchsten sachlichen wie theoretischen Gewichts ${ }^{20}$. Im Hinblick auf die Frage nach der Rationalität des Bürokratietypus aber hat das zur Konsequenz: auch auf diesem Felde wird

19 Albrow (1972: 74 ff.) stützt sich vor allem auf den Redebeitrag Max Webers auf der Wiener Tagung des Vereins für Sozialpolitik von 1909 (1924: $412 \mathrm{ff}$.). Weber hat hier das ,hochmoralische" preuBisch-deutsche Beamtentum konfrontiert mit den korruptionsanfälligen Bürokratien der ,demokratisch regierten Länder* (Frankreich, USA, England) und dabei das ,,business-Beamtentum" der letzteren für in Sachen (wörtlich), efficiency" überlegen erklärt. Dabei muß man allerdings sehen, daß bei diesen Bemerkungen Webers durchaus die politische Absicht der ,Entmythologisierung' der preußischen Bürokratie mit im Spiel war (Beetham 1974: 64 f.).

20 So sagt Weber mit Bezug auf die ProtestantismusStudie: „Wenn zu irgend etwas, so möchte dieser Aufsatz dazu beitragen, den nur scheinbar eindeutigen Begriff des ,Rationalen' in seiner Vielseitigkeit aufzudecken" (1920: 35, Anm. 1). man von der Mehrdeutigkeit des Rationalen auszugehen haben; will man also der bürokratischen Rationalität im Sinne Max Webers auf die Spur kommen, so hat man dieser Mehrdeutigkeit nicht nur Rechnung zu tragen, sondern muß sie zum Thema machen.

Das kann hier in angemessener Form natürlich nicht mehr geschehen; wohl aber sollen nunmehr zwei, m.E. allerdings zentrale Bedeutungsschwerpunkte der bürokratischen Rationalitat, wie sie Max Weber verstand, abschließend noch skizzenhaft zur Sprache kommen. Es sollte nicht verwundern, daß dabei der Zweckrationalität kaum besonderes Gewicht beigemessen wird; ich bin ähnlich wie Constans Seyfarth (1979) der Auffassung, daß der Zweckrationalität innerhalb der Weberschen Rationalitatskonzeption nicht der Stellenwert zukommt, den man ihr nach der Lekture allein der methodologischen Schriften beimessen möchte.

Der erste hier zu behandelnde Bedeutungsschwerpunkt zielt auf ein Verständnis des Rationalen und der Rationalisierung im Sinne von ,Konsequenz', von ,Systematisierung, Methodisierung und Vereinheitlichung ' (etwa Weber 1920: 512, 537). Was unter diesen Vorzeichen das moderne bürokratische Verwaltungshandeln in exklusiver Weise kennzeichnet, ist gewissermaßen die Perfektionierung von Herrschaft: der herrschaftssoziologische Zugriff Webers ist gewählt von der Durchsetzungs- und Fügsamkeitsproblematik her ${ }^{21}$; es geht um Befehl und Gehorsam. Gehorsam, nämlich Disziplin (1964, I: 38), aber ,steht' im spezifischen Kontext der Bürokratie in einem historisch nie gekannten Ausmaß gewissermaßen pauschal und kombiniert mit fachlicher Schulung ,zur Verfugung (ähnlich Luhmann 1971: 106); dies bedeutet (fur den ,Herrn') die strukturelle Chance, das Handeln des, Verwaltungsstabs" lückenlos, auf der ganzen Linie primär mittels formaler Regeln ,programmieren", mithin festlegen und binden zu können. Noch der geringfügigste ,Verwaltungsakt" ist dadurch tangiert; ,,entscheidend ist ...., daß prinzipiell hinter jeder Tat echt bitrokratischer Verwaltung ein System rational diskutabler ,Gründe;, d.h. entweder: Subsum-

$21 \mathrm{Vgl}$. die Webersche Machtdefinition (1964, I: 38), vor allem aber seine höchst wichtigen, Voruberlegungen' dazu (1964, II: 691 ff.); des näheren Tyrell 1980 . 
tion unter Normen, oder: Abwägung von Zwekken und Mitteln steht" (Weber 1964, II: 721). Solche Rationalisierung bedeutet ersichtlich zugleich auch ,Intellektualisierung", was hier aber außer Acht bleiben kann. Entscheidend ist hier stattdessen die Lückenlosigkeit, Konsequenz und Reichweite, mit der es im bürokratischen Kontext gelingt, das Handeln des Verwaltungsstabs festzulegen und zu steuern.

Dem entspricht im bürokratischen Verwaltungshandeln jene Kombination von ,rationaler", nämlich methodischer Disziplin (1964, Il: 866 f.), strikter Sachlichkeit/,Unpersönlichkeit * (hierzu jetzt Gabriel 1979: 32 ff.) und ,fachlicher Eingeschultheit". Der bürokratische Rationalisierungsproze $ß$ ist wesentlich $\operatorname{der}$ Prozeß der strukturellen Freisetzung und Ausdifferenzierung dieses auf Konsequenz gebauten Verhaltenssyndroms. Auf die Art kann dann die Bin. dung des Verwaltungshandelns, auf der ganzen Linie“ gelingen ohne das ,Risiko“ der ,Kontamination durch abweichende und zusätzliche Motive" (Offe 1974: 334), also ,ohne allen Einfluß persönlicher Motive oder gefühlsmäßiger Einflüsse, frei von Willkür und Unberechenbarkeiten" (Weber 1973: 476) und auf dem Niveau gehobener Schulung und Fachqualifikation.

Nicht (oder doch bestenfalls erst sekundär) um den ,Output' des Verwaltungshandelns geht es Weber, sondern um das präzise, eindeutige, kontinuierliche, immer regelgebundene, mithin berechenbare ,Funktionieren' der bürokratischen Abwicklung von - sachlich vergleichsweise anspruchsvollen, Fachqualifikation erfordernden - Amtsgeschäften (etwa 1964, II: 717). Im wesentlichen darauf zielen die immer neuen technisch-instrumentellen Analogien, die schon angesprochenen Kennzeichnungen der Bürokratie als ,Maschine, Apparat, Mechanismus" usw.. Wenn man hier schon von ,Effizienz' sprechen will, so muß man sie auf die Präzision des ,regelgebundenen Betriebs' der Bürokratie beziehen; in dieser koinzidieren die anspruchsvolle Sachorientierung der Amtsausübung einerseits und das auf Disziplin gebaute und damit gänzlich ,entlastete" Funktionieren des Durchsetzungsmechanismus andererseits.

Die zweite Bedeutungsvariante des Rationalen, auf die hier noch eingegangen werden soll, hat einen spezifisch voluntaristischen und zugleich auf Innovation setzenden Akzent. Formeln, die Weber dafür bereithält, sind die der, bewußten Neuschöpfung، (1964, I: 26 f.), des ,rational Geschaffenen' (1964, II: 739) oder des ,absichtsvoll neu Schaffens' (1964, I: 167). Solche Tendenz zum ,, bewußt rational Geschaffenen“ (1964, I: 12) ist für Weber ein ganz wesentliches Kennzeichen der Handlungstypik, die dem okzidentalen Rationalismus spezifisch ist. Sie bedeutet, daß - gesellschaftsstrukturell verankert - , das praktisch relevante Handeln" in nie gekanntem Maße machbar, änderbar-innovierbar und explizit entscheidbar wird.

Gerade das rückt nun den Rationalismus der Moderne in den nachdrücklichsten Gegensatz zu allem Traditionalismus, denn diesem, wie ihn Weber verstand, ist gerade die Blockierung jeder Änderung oder Neuschöpfung spezifisch. Die änderungsabgeneigte kollektive Mentalität des Traditionalismus kann gestützt oder noch forciert werden durch, Interessenkonstellationen'; aber Weber verweist darüberhinaus noch auf einen anderen ,Verstärker': ,Angst vor magischen Nachteilen verstärkte die psychische Hemmung gegenüber jeder Ânderung eingelebter Gepflogenheiten des Handelns" (Weber 1964, I: 26); für eben diesen Sachverhalt hat er an anderer Stelle (1923: $302 \mathrm{f}$.) den Begriff der, magischen Stereotypisierung des Handelns: Sieht man näher hin, dann durchzieht, wie man schnell bemerken wird, dieses bislang so gut wie ,unentdeckte " und durchaus theoretisch zu nennende Konzept nahezu Webers gesamte Soziologie der, vorrationalistischen Epochen" (1964, I: 182). Der universalhistorische Rationalisierungsprozen bedeutet daraufhin die epochale Freisetzung des Handelns von ,magischer Stereotypisierung (teils auch: Stereotypierung) und traditionalistischer Gebundenheit"; er bedeutet ,Entbindung' und damit zugleich Freigabe einer relativen Beliebigkeit des Handelns, genauer: eines änderungswilligen und innovativen Handelns bei variablen $Z$ wecken.

Eben daran aber haben nun die Handlungssphären von Herrschaft und Recht ihren ganz entscheidenden Anteil; ja, diese Handlungstypik ist geradezu verkörpert in dem rationallegalen Legitimitätstypus, wie ihn die Webersche Herrschaftssoziologie präsentiert. Bekannt- 
lich basiert die Legitimität der rational-legalen Herrschaft auf der ,Grundvorstellung . . ., daß durch formal korrekt gewillkurte Satzung beliebiges Recht geschaffen und abgeändert werden könne" (1973: 475; auch 1964, I: 26 f., 160 f.). Hier ist die ,Gemachtheit", nämlich: ,Gesatztheit' und Änderbarkeit des Rechts institutionalisiertes Prinzip, und, daß hier in diesem Sinne der Rechtsmechanismus auf Dauer dem zweckorientierten innovativen Zugriff zur Verfügung und zur Disposition steht, bringt den legalen Herrschaftstypus in den schärfsten Gegensatz zum traditionalen, welcher ,tatsächliche Neuschöpfungen“ ,nur als von jeher geltend und nur durch ,Weistum' erkannt legitimieren" konnte $(1964$, l: 167 f.). Es versteht sich angesichts dessen von selbst, daß für die Webersche Rechtssoziologie die Rationalisierung des Rechts wesentlich (auch) dessen Positivierung bedeutete ${ }^{22}$, also im Effekt: ,die zunehmende Wertung des jeweils geltenden Rechts als eines rationalen, daher jederzeit zweckrational umzuschaffenden, jeder inhaltlichen Heiligkeit entbehrenden, technischen Apparats" (1964, I: 656).

Wie auch Winckelmann (1964: 61 f.) feststellt, sind für Weber die rational-legale und die bürokratische Herrschaft nicht einfach deckungsgleich; vielmehr ist die letztere ein Sonderfall der ersteren ${ }^{23}$, wenngleich natürlich der prominenteste und von Weber am breitesten behandelte Fall. Diese Subsumtion des, regelgebundenen Betriebs' der Bürokratie unter den rational-legalen Legitimitätstypus will nun vor allem besagen, daß für die bürokratischen Verfahrensregeln und ,Normen', sofern sie nicht

$22 \mathrm{Vgl}$. etwa auch Abramowski 1966: $140 \mathrm{f}$, $144 \mathrm{ff}$., und Beetham 1974: 68. Webers, Schaffens'-Terminologie scheint primär dem Rechtskontext entnommen $z u$ sein; siehe dazu und $z u$ dem historisch sehr frühen Fall des klassischen Athen, in dem die Positivierung des Rechts, die Durchsetzung ,der Auffassung des Rechts als einer rationalen Schöpfung" nahezu vollzogen war, Weber 1964, II: $993 \mathrm{f}$.

23 ,Die Bürokratie ist nicht der einzige Typus legaler Herrschaft. Das Turnus-, Los- und Wahlbeamtentum, die Parlaments- und Komiteeverwaltung und alle Arten kollegialer Herrschafts- und Verwaltungskörper fallen darunter, falls ihre Kompetenz auf gesatzten Regeln beruht und die Ausübung des Herrschaftsrechtes dem Typus legalen Verwaltens entspricht" (Weber 1973: 477). ohnehin Rechtsqualität haben, gilt, was auch fur das positivierte Recht gilt: sie sind absichtsvoll und bewußt ,gemacht", sind, ,rational geschaffen, appellieren an den Sinn für abstrakte Legalität, ruhen auf technischer Einschulung" (1964, II: 739). Und damit zugleich: diese gesatzten ,technischen Regeln' und ,Normen (1964, I: 161) sind nach Zweckmäßigkeitsgesichtspunkten jederzeit änderbar, ,umschaffbar'; der spezifisch, technische' Charakter gilt für sie erst recht, sind sie doch forciert Gegenstand rationaler Entscheidungstätigkeit und rationalen Disponierens. Die so verstandene technische Verfügung über die Regeln, die das bürokratische Handeln steuern, ist eines der ganz entscheidenden Momente an der Rationalität der Bürokratie, wie sie Weber verstand und allen Formen traditionaler Herrschaft und magisch stereotypisierter Gebundenheit gegenüberstellte.

Nun führt aber der explizite Gegensatz von Stereotypisierung ' und ,Rationalisierung' (Weber 1964, II: 767) noch weiter; er bezieht sich keineswegs nur auf das Reglement der Bürokratie; ebenso zielt er - modern gesprochen - auf die Stellen. Die traditionale Herrschaft neigt durchweg zur, Stereotypisierung der Ämterverfassung“ (1964, II: $758 \mathrm{ff}$ ), - zu einem ,Gebilde also, welches starr, neuen Aufgaben nicht anpassungsfahig, der abstrakten Reglementierung unzugänglich, ein charakteristisches Gegenbild gegen die zweckvoll abstrakt geordneten und gegebenenfalls jederzeit neu zu ordnenden "Kompetenzen' der bürokratischen Struktur darstellt" (1964, II: 769). Die Änderbarkeit der Ämterorganisation oder eben der Stellen war für Weber ersichtlich ein wesentlicher Punkt, und das Gleiche gilt im Hinblick auf das Personal. Die für die moderne Bürokratie immer wieder betonte, Trennung von Amt und Person", insbesondere die Nichtappropriation der Ämter durch das Personal bedeutet ja wesentlich die ,freie Hand“ der Spitze der Bürokratie diesem gegenüber. Dabei hatte Weber insbesondere die Rekrutierung des Personals im Auge: ,Kontrakts-Anstellung, also freie Auslese, ist der modernen Bureaukratie wesentlich" (1964, I: 163). Und das Gleiche, nämlich ,Freiheit der Arbeiterauslese" gilt, wie Weber (1964, I: 121 ff.) in kaum zu uberbietender Schärfe und Knappheit dargelegt hat, für den modernen kapitalistischen Betrieb. Ein Unterschied liegt allerdings in der ,Lebenslänglich- 
keit der Stellung' des Beamten. Diese ,gilt jedoch nicht, wie in vielen Herrschaftsformen der Vergangenheit, als ein ,Besitzrecht' des Beamten am Amt"; vielmehr zielt sie auf ,eine Garantie für die streng sachliche, von persönlichen Rücksichten freie Ableistung der betreffenden spezifischen Amtspflicht" (Weber 1964, II: 708). Der Möglichkeit der Versetzung und vor allem der, vertikalen Mobilität" des Personals innerhalb der Amtshierarchie ist aber auch damit natürlich kein $\mathrm{Ab}$ bruch getan.

Zieht man all dies zusammen, so ergibt sich in der Tendenz genau das, was der neueren systemtheoretischen Organisationssoziologie im Hinblick auf ,Organisation' konstitutiv ist, nämlich die prinzipielle Änderbarkeit und Variierbarkeit der Regeln, der Stellen und des Personals (etwa Luhmann 1975: 40 ff.). Eben darin hat Max Weber einen ganz entscheidenden rationalen Zug der modernen Bürokratie gesehen.

\section{Literatur:}

Abramowski, G., 1966: Das Geschichtsbild Max Webers: Universalgeschichte am Leitfaden des okzidentalen Rationalisierungsprozesses. Stuttgart.

Albrow, M., 1972: Bürokratie. München.

Bader, V.M./Berger, J./Ganssmann, H./Knesebeck, J.v.d., 1976: Einfuihrung in die Gesellschaftstheorie: Gesellschaft, Wirtschaft und Staat bei Marx und Weber. 2 Bde.. Frankfurt u. New York.

Beetham, D., 1974: Max Weber and the Theory of Modern Politics. London.

Bendix, R., 1947: Bureaucracy: The Problem and its Setting. American Sociological Review 12: 493507.

Bendix, R., 1964: Max Weber: Das Werk (Darstellung, Analyse, Ergebnisse). München.

Bendix, R., 1965: Die vergleichende Analyse historischer Wandlungen. Kölner Zeitschrift für Soziologie und Sozialpsychologie 17: 429-446.

Berger, J., 1978: Intersubjektive Sinnkonstitution und Sozialstruktur: Zur Kritik handlungstheoretischer Ansätze. Zeitschrift für Soziologie 7: 327-334.

Diamant, A., 1962: The Bureaucratic Model: Max Weber Rejected, Rediscovered, Reformed. S. 59-96 in: F. Heady u. S.L. Stokes, Hrsg., Papers in Public Administration. Ann Arbor.

Gabriel, K., 1974: Organisation und Legitimation: Die Selbststeuerungsimperative der Organisation und das Problem der Legitimation. Zeitschrift für Soziologie 3: 339-355.
Gabriel, K., 1976: Organisation und sozialer Wandel. S. 301-324 in: G. Büschges, Hrsg., Organisation und Herrschaft: Klassische und moderne Studientexte zur sozialwissenschaftlichen Organisationstheorie. Reinbek.

Gabriel, K., 1979: Analysen der Organisationsgesellschaft: Ein kritischer Vergleich der Gesellschaftstheorien Max Webers, Niklas Luhmanns und der phänomenologischen Soziologie. Frankfurt/M u. New York.

Grunow, D./Hegner, F., 1977: Von der Bürokratiekritik zur Analyse des Netzes bürokratischer Organisationen. S. 45-79 in: Th. Leuenberger/ K.H. Ruffmann, Hrsg., Bürokratie: Motor oder Bremse der Entwicklung? Bern, Frankfurt/M. u. Las Vegas.

Hintze, O., 1964: Der Beamtenstand. S. 66-125 in: O. Hintze, Soziologie und Geschichte: Gesammelte Abhandlungen zur Soziologie. Politik und Theorie der Geschichte (Gesammelte Abhandlungen II). 2. erw. Aufl.. Göttingen.

Kieser, A./Kubicek, H., 1978: Organisationstheorie I: Wissenschaftstheoretische und kritische Analyse klassischer Ansätze. Stuttgart u.a.

Kröpp, W., 1979: Zur Grundlegung des Interaktionismus: Soziologie als Wirklichkeitswissenschaft. Diss. Bielefeld.

Lindenlaub, D., 1967: Richtungskämpfe im Verein für Sozialpolitik: Wissenschaft und Sozialpolitik im Kaiserreich vornehmlich vom Beginn des, Neuen Kurses' bis zum Ausbruch des ersten Weltkrieges (1890-1914). 2 Bde. Wiesbaden.

Lipp, W., 1978: Bürokratische, partizipative und Kaderorganisation als Instrumente sozialer Steuerung: Typenanalyse und Leistungsvergleich. Die Verwaltung $11: 3-25$.

Luhmann, N., 1971: Zweck - Herrschaft - System: Grundbegriffe und Prämissen Max Webers. S. 90112 in: N. Luhmann, Politische Planung: Aufsätze zur Soziologie von Politik und Verwaltung. Opladen.

Luhmann, N., 1975: Allgemeine Theorie organisierter Sozialsysteme. S. 39-50 in: N. Luhmann, Soziologische Aufklärung 2: Aufsätze zur Theorie der Gesellschaft. Opladen.

Mayntz, R., Hrsg., 1971a: Bürokratische Organisation. Köln u. Berlin.

Mayntz, R., 1971b: Max Webers Idealtypus der Bürokratie und die Organisationssoziologie. S. 27-35 in: R. Mayntz, Hrsg., Bürokratische Organisation. Köln u. Berlin.

Mommsen, W.J., 1974a: Max Weber: Gesellschaft, Politik und Geschichte. Frankfurt/M.

Mommsen, W.J., 1974b: The Age of Bureaucracy: Perspectives on the Political Sociology of Max Weber. New York u.a..

Müller, W., 1973: Die Relativierung des bürokratischen Modells und die situative Organisation. Kölner Zeitschrift für Soziologie und Sozialpsychologie 25: 719-749.

Offe, C., 1974: Rationalitätskriterien und Funktionsprobleme politisch-administrativen Handelns. Leviathan 2: 333-345.

Pfeiffer, D.K., 1976: Organisationssoziologie: Eine Einfuihrung. Stuttgart u.a. 
Prewo, R., 1979: Max Webers Wissenschaftsprogramm: Versuch einer methodischen Neuerschließung. Frankfurt/M.

Roth, G., 1968: Introduction. S. XXVII-CIV in: G. Roth/C. Wittich (Hrsg.), Max Weber, Economy and Society: An Outline of Interpretative Sociology. Bd. I. New York.

Schluchter, W., 1972: Aspekte bürokratischer Herrschaft: Studien zur Interpretation der fortschreitenden Industriegesellschaft. München.

Schmoller, G., 1894: Einleitung: Über Behördenorganisation, Amtswesen und Beamtenthum im Allgemeinen und speciell in Deutschland und Preußen bis zum Jahre 1713. S. 13-143 in: Acta Borussica. Die Behördenorganisation und die Allgemeine Staatsverwaltung Preußens im 18. Jahrhundert. 1. Bd. Berlin.

Schmoller, G., 1898: Der deutsche Beamtenstaat vom 16. bis 18. Jahrhundert. S. $289-313$ in: $G$. Schmoller, Umrisse und Untersuchungen zur Verfassungs-, Verwaltungs- und Wirtschaftsgeschichte besonders des Preußischen Staates im 17. und 18. Jahrhundert. Leipzig.

Seyfarth, C., 1979: Alltag und Charisma bei Max Weber: Eine Studie zur Grundlegung der, verstehenden" Soziologie. S. 155-177 in: W.M. Sprondel/ R. Grathoff, Hrsg., Alfred Schütz und die Idee des Alltags in den Sozialwissenschaften. Stuttgart.

Tyrell, H., 1980: Gewalt, Zwang und die Institutionalisierung von Herrschaft: Versuch einer Neuinterpretation von Max Webers Herrschaftsbegriff. S. 5992 in: R. Pohlmann, Hrsg., Person und Institution: H. Schelsky gewidmet. Würzburg.
Weber, M., 1920: Gesammelte Aufsätze zur Religionssoziologie. Bd. I.. Tübingen.

Weber, M., 1923: Wirtschaftsgeschichte: Abriß der universalen Sozial- und Wirtschaftsgeschichte. Hrsg. v. S. Hellmann u. M. Palyi. München.

Weber, M., 1924: Gesammelte Aufsätze zur Soziologie und Sozialpolitik. Tübingen.

Weber, M., 1964: Wirtschaft und Gesellschaft: Grundriß der verstehenden Soziologie. Studienausgabe. 2 Bde.. Hrsg. v. J. Winckelmann. Köln u. Berlin

Weber, M., 1971a: Parlament und Regierung im neugeordneten Deutschland: Zur politischen Kritik des Beamtentums und Parteiwesens. S. $306-443$ in: M. Weber, Gesammelte politische Schriften. Hrsg. v. J. Winckelmann. 3. erneut vermehrte Aufl.. Tübingen.

Weber, M., 1971 b: Politik als Beruf. S. 505-560 in: M. Weber, Gesammelte politische Schriften. Hrsg. v. J. Winckelmann. 3. erneut vermehrte Aufl., Tübingen.

Weber, M., 1973: Gesammelte Aufsätze zur Wissenschaftslehre. Hrsg. v. J. Winckelmann. 4. durchges. Aufl.. Tübingen.

Weiß, J., 1975: Max Webers Grundlegung der Soziologie: Eine Einführung. München.

Winckelmann, J., 1964: Max Webers historische und soziologische Verwaltungsforschung. Annali della Fondazione per la Storia Amministrativa 1: 27-67.

Zingerle, A., 1972: Max Weber und China: Herrschaftsund religionssoziologische Grundlagen zum Wandel der chinesischen Gesellschaft. Berlin. 